\title{
APPROXIMATION OF NONLINEAR WAVE EQUATIONS WITH NONSTANDARD ANISOTROPIC GROWTH CONDITIONS
}

\author{
JONAS HAEHNLE AND ANDREAS PROHL
}

\begin{abstract}
Weak solutions for nonlinear wave equations involving the $p(\mathbf{x})$ Laplacian, for $p: \Omega \rightarrow(1, \infty)$ are constructed as appropriate limits of solutions of an implicit finite element discretization of the problem. A simple fixed-point scheme with appropriate stopping criteria is proposed to conclude convergence for all discretization, regularization, perturbation, and stopping parameters tending to zero. Computational experiments are included to motivate interesting dynamics, such as blowup, and asymptotic decay behavior.
\end{abstract}

\section{INTRODUCTION}

Let $T>0$, and $\Omega \subset \mathbb{R}^{d}, d \geq 1$ be a bounded Lipschitz domain. Consider $p \in C(\bar{\Omega},(1, \infty))$, such that

$$
|p(\mathbf{x})-p(\mathbf{y})| \leq \frac{c}{|\ln | \mathbf{x}-\mathbf{y}||} \quad \forall|\mathbf{x}-\mathbf{y}|<\frac{1}{2},
$$

for some $c>0$. Suppose that $f: \Omega \times \mathbb{R} \rightarrow \mathbb{R}$ is continuous, with suitable growth conditions, and $F(\mathbf{x}, u)=\int_{0}^{u} f(\mathbf{x}, s)$ d $s$. Given $\left(u_{0}, v_{0}\right) \in W_{0}^{1, p(\mathbf{x})}(\Omega) \times L^{2}(\Omega)$, we seek (global weak) solutions $u:(0, T) \times \Omega \rightarrow \mathbb{R}$, such that $(\alpha \geq 0)$

$$
\begin{aligned}
u_{t t}-\operatorname{div}\left(|\nabla u|^{p(\mathbf{x})-2} \nabla u\right)-\alpha \Delta u_{t}+f(\mathbf{x}, u)=0 & \text { in } \Omega_{T}:=(0, T] \times \Omega, \\
(1.2) \quad u(0, \cdot)=u_{0}, \quad u_{t}(0, \cdot)=v_{0} & \text { in } \Omega, \\
u=0 & \text { on } \partial \Omega_{T}:=(0, T] \times \partial \Omega .
\end{aligned}
$$

This is a prototype problem with nonstandard $p(\mathbf{x})$-growth condition, and energy functional

$$
E_{p}[u, v]=\int_{\Omega}\left[\frac{1}{p(\mathbf{x})}|\nabla u|^{p(\mathbf{x})}+\frac{1}{2}|v|^{2}+F(\mathbf{x}, u)\right] \mathrm{d} \mathbf{x} \quad \text { for } p \in C(\bar{\Omega} ;(1, \infty)),
$$

where the classical solutions to (1.2) satisfy

$$
E_{p}\left[u(t, \cdot), u_{t}(t, \cdot)\right]+\alpha \int_{\Omega_{T}}\left|\nabla u_{t}\right|^{2} \mathrm{~d} \mathbf{x} \mathrm{d} t=E_{p}\left[u_{0}, v_{0}\right] \quad \forall t \geq 0 .
$$

One motivation comes from studying evolutionary problems with nonstandard anisotropic growth conditions, which may e.g. provide further insight into the behavior of solutions of semi-/quasilinear wave equations with critical (de-)focusing nonlinearities, such as finite time blow-up behavior 4, 22, 8, 27, 14, 16, 7, 32, 17, 18, decay behavior of global solutions [6, 26, 5, 3], relevancy of weak and/vs. strong

Received by the editor December 19, 2007 and, in revised form, July 23, 2008.

2000 Mathematics Subject Classification. Primary 35K55, 65M12, 65M15.

(C)2009 American Mathematical Society 
damping in this context, and/or dependence of solutions on initial data of small, finite, or infinite energies [24, 14. A possible physical motivation for (1.2) are models from viscoelasticity, where (1.2) for $2 \leq p \equiv$ const is the subject of several studies; see e.g. [15, 28].

Functionals with variable exponents (1.3) are currently the subject of intensive research, and analytical studies of variational problems with nonstandard $p(\mathbf{x})$ growth have led to interesting results, and are still rapidly developing; see e.g. [11, 2, 12, 13, 2, 30, 10. However, it is only recently that related parabolic equations with anisotropic nonstandard growth conditions have been studied, both analytically [1] and numerically [29, where the latter work includes computational studies to motivate decay behavior, or blowup of solutions for supercritical nonlinearities. The goal of this work is to extend this program to the nonlinear wave equation (1.2). In particular, we

(1) construct a convergent finite element based scheme: A fully practical discretization is provided, which includes a fixed-point strategy to solve nonlinear algebraic problems at each iteration step, in combination with an appropriate stopping criterion. In particular, the fixed-point algorithm requires a regularization $\Delta_{p(\mathbf{x})}^{\delta(\mathbf{x})} u:=\operatorname{div}\left(\left[|\nabla u|^{2}+\delta(\mathbf{x})\right]^{\frac{p(\mathbf{x})-2}{2}} \nabla u\right)$, for $\delta \in L^{\infty}(\Omega,(0,1])$ of $\Delta_{p(\mathbf{x})} u:=\operatorname{div}\left(|\nabla u|^{p(\mathbf{x})-2} \nabla u\right)$ in (1.2) to validate a contraction property for all $p \in C(\bar{\Omega},[2, \infty))$. Overall convergence of iterates to a weak solution of (1.2) in the sense of Definition 2.1 will be shown for all discretization, perturbation, regularization, and thresholding parameters tending to zero.

(2) perturb the numerical scheme: Since a complicated $p: \Omega \rightarrow[2, \infty)$ crucially affects numerical integration, approximations $p_{\epsilon} \in C(\Omega,[2, \infty))$ might be useful; we verify that for $p_{\epsilon} \downarrow p$ simultaneously to other convergences in item (1), iterates of the numerical scheme (see Scheme A, and Algorithm $\mathrm{A}_{1}$ below) converge to weak solutions of (1.2).

(3) computationally study the qualitative behavior of solutions: Scheme A and Algorithm $\mathrm{A}_{1}$ are convergent discretizations, in the sense that subsequences of solutions converge to weak solutions of (1.2). This theoretical background justifies computational studies to motivate interesting behaviors of weak solutions of (1.2), such as asymptotic decay properties for subcritical nonlinearities. Moreover, computational experiments are provided for situations which cannot be covered theoretically so far, such as $\alpha=0$, and values $p_{-}:=\inf _{\Omega} p<2$, large or infinite initial energies, and supercritical involved nonlinearities.

The remainder of this work is organized as follows. In Section 2, we recall useful properties of the Orlicz spaces $L^{p(\mathbf{x})}$ and $W^{m, p(\mathbf{x})}$, and define weak solutions of (1.2). In Section 3, we propose an implicit, regularized finite element discretization of (1.2) (Scheme A) and validate solvability, and obtain a discrete version of the energy identity (1.4) for iterates of Scheme A $\left(\alpha \geq 0\right.$ and $\left.p_{-}>0\right)$. Subsequence convergence of iterates to weak solutions in the sense of Definition 2.1 for (independently) vanishing discretization and regularization parameters is stated in Theorem 3.1, for $\alpha>0$, and $p_{-} \geq 2$. This result is achieved for initial data of finite energy, and functions $f$ in (1.2) which satisfy the asymptotic growth condition (2.3), for $1<\gamma<p_{-}$. 
Solving nonlinear problems in Scheme A requires an iterative procedure; in Section 4, we discuss how the goal to validate a discrete energy law interferes with the goal to validate a contraction property at this point. The fixed-point algorithm, Algorithm $\mathrm{A}_{1}$, together with a stopping criterion, is proposed, and overall convergence of iterates of this fully practical scheme for all discretization, regularization, and thresholding parameters tending to zero is stated in Theorem 4.1. Computational examples addressing both issues related to the given numerical schemes and qualitative behaviors of solutions are reported in Section 5 .

\section{Preliminaries}

Below, unless explicitly stated, always let $\Omega \subset \mathbb{R}^{d}$, for $d=2,3$, be a bounded Lipschitz domain, and let $p, p_{\epsilon} \in C(\bar{\Omega},(1, \infty))$ satisfy (1.1). The material presented in Section 2.1] can be found in [13, 33].

2.1. Spaces $L^{p(\mathbf{x})}(\Omega)$ and $W^{m, p(\mathbf{x})}(\Omega)$. Let $p \in C(\bar{\Omega} ;(1, \infty))$ be given, with $p \in$ $\left[p_{-}, p_{+}\right], p_{+}:=\sup _{\Omega} p$, and let (1.1) be valid. We define the generalized Lebesgue space

$L^{p(\mathbf{x})}(\Omega):=\{u: \Omega \rightarrow \mathbb{R}: u$ is a measurable real-valued function,

$$
\left.\rho_{p(\mathbf{x})}(\lambda u)<\infty \text { for } \lambda>0\right\},
$$

where $\rho_{p(\mathbf{x})}(u)=\int_{\Omega}|u|^{p(\mathbf{x})} \mathrm{d} \mathbf{x}$ is called the modular. We introduce the so-called Luxemburg norm on $L^{p(\mathbf{x})}(\Omega)$,

$$
\|u\|_{L^{p(\mathbf{x})}}:=\inf \left\{\lambda>0: \rho_{p(\mathbf{x})}\left(\frac{u}{\lambda}\right) \leq 1\right\} .
$$

If $p$ is constant, then the variable exponent Lebesgue spaces coincide with the classical Lebesgue space. For all $u \in L^{p(\mathbf{x})}(\Omega)$, the following holds:

$$
\min \left\{\|u\|_{L^{p(\mathbf{x})}}^{p_{-}},\|u\|_{L^{p(\mathbf{x})}}^{p_{+}}\right\} \leq \rho_{p(\mathbf{x})}(u) \leq \max \left\{\|u\|_{L^{p(\mathbf{x})}}^{p_{-}},\|u\|_{L^{p(\mathbf{x})}}^{p_{+}}\right\} .
$$

The tuple $\left(L^{p(\mathbf{x})}(\Omega),\|\cdot\|_{L^{p(\mathbf{x})(\Omega)}}\right)$ is a separable Banach space, and its conjugate space is $L^{q(\mathbf{x})}(\Omega)$, for $\frac{1}{q(\mathbf{x})}+\frac{1}{p(\mathbf{x})}=1$. Given $u \in L^{p(\mathbf{x})}(\Omega)$ and $v \in L^{q(\mathbf{x})}(\Omega)$, it follows that

$$
\left|\int_{\Omega} u v \mathrm{~d} \mathbf{x}\right| \leq\left(\frac{1}{p_{-}}+\frac{1}{q_{-}}\right)\|u\|_{L^{p(\mathbf{x})}(\Omega)}\|v\|_{L^{q(\mathbf{x})}(\Omega)} .
$$

If $p_{1}, p_{2} \in C(\bar{\Omega},(1, \infty))$, such that $p_{1}(\mathbf{x}) \leq p_{2}(\mathbf{x})$ in $\bar{\Omega}$, then $L^{p_{2}(\mathbf{x})}(\Omega) \subset L^{p_{1}(\mathbf{x})}(\Omega)$, and the embedding is continuous.

The (separable, reflexive) Banach space $W_{0}^{1, p(\mathbf{x})}(\Omega)$ is defined by

$$
W_{0}^{1, p(\mathbf{x})}(\Omega):=\left\{u \in L^{p(\mathbf{x})}:|\nabla u| \in L^{p(\mathbf{x})}(\Omega), u=0 \text { on } \partial \Omega\right\},
$$

which is endowed with the norm

$$
\|u\|_{W_{0}^{1, p(\mathbf{x})}}:=\|u\|_{L^{p(\mathbf{x})}}+\|\nabla u\|_{L^{p(\mathbf{x})}} .
$$

An equivalent norm of $W_{0}^{1, p(\mathbf{x})}(\Omega)$ is given by $\|\nabla u\|_{L^{p(\mathbf{x})}(\Omega)}$. We have that $C_{0}^{\infty}(\bar{\Omega})$ is dense in $W_{0}^{1, p(\mathbf{x})}(\Omega)$. The embedding $W_{0}^{1, p(\mathbf{x})}(\Omega) \subset L^{q(\mathbf{x})}(\Omega)$ is continuous and compact if

$$
1<q(\mathbf{x}) \leq \sup _{\Omega} q(\mathbf{x})<\inf _{\Omega} p_{*}(\mathbf{x}) \quad \text { with } p_{*}(\mathbf{x})=\left\{\begin{array}{rr}
\frac{d p(\mathbf{x})}{d-p(\mathbf{x})}, & p(\mathbf{x})<d, \\
\infty, & p(\mathbf{x})>d .
\end{array}\right.
$$


Moreover,

$$
\|u\|_{L^{p(\mathbf{x})}} \leq C\|\nabla u\|_{L^{p(\mathbf{x})}} \quad \forall u \in W_{0}^{1, p(\mathbf{x})}(\Omega) .
$$

We define the $p(\mathbf{x})$-Laplace operator $-\Delta_{p(\mathbf{x})} u:=-\operatorname{div}\left(|\nabla u|^{p(\mathbf{x})-2} \nabla u\right)$; it is the derivative of the strictly convex functional $E_{p(\mathbf{x})}: W_{0}^{1, p(\mathbf{x})}(\Omega) \rightarrow \mathbb{R}$ given in (1.3). The mapping $-\Delta_{p(\mathbf{x})}: W_{0}^{1, p(\mathbf{x})}(\Omega) \rightarrow\left(W_{0}^{1, p(\mathbf{x})}(\Omega)\right)^{*}$ is continuous, bounded, and strictly monotone [11].

2.2. Weak solution of (1.2). Let $f: \Omega \rightarrow \mathbb{R}$ be a continuous function satisfying the growth condition

$$
|f(\mathbf{x}, s)| \leq h_{f}(\mathbf{x})+C_{1}|s|^{\gamma-1} \quad \forall(\mathbf{x}, s) \in \Omega \times \mathbb{R},
$$

for some $\gamma>1$, and $h_{f} \in L^{\infty}\left(\Omega ; \mathbb{R}^{+}\right)$. In the following, we define weak solutions to (1.2).

Definition 2.1. Fix $T>0$, and let $\Omega \subset \mathbb{R}^{d}$ be a bounded Lipschitz domain. Let $p \in C(\bar{\Omega},(1, \infty))$ be such that (1.1) holds, and $\left(u_{0}, v_{0}\right) \in W_{0}^{1, p(\mathbf{x})}(\Omega) \times L^{2}(\Omega)$. Then $u: \Omega_{T} \rightarrow \mathbb{R}$ is called a weak solution to (1.2) $(\alpha>0)$ if

(i) $u \in L^{\infty}\left(0, T ; W_{0}^{1, p(\mathbf{x})}(\Omega)\right) \cap W^{1, \infty}\left(0, T ; L^{2}(\Omega)\right) \cap W^{1,2}\left(0, T ; W_{0}^{1,2}(\Omega)\right)$,

(ii) initial data are attained, i.e., for $t \rightarrow 0$,

$$
u(t, \cdot) \rightarrow u_{0} \quad \text { in } W^{1, p(\mathbf{x})}(\Omega), \quad u_{t}(t, \cdot) \rightarrow v_{0} \quad \text { in } L^{2}(\Omega),
$$

(iii) for all $\xi \in C_{0}^{\infty}([0, T) \times \Omega)$ it follows that

$$
\begin{aligned}
\int_{\Omega_{T}}\left[-u_{t} \xi_{t}+|\nabla u|^{p(\mathbf{x})-2} \nabla u\right. & \left.\cdot \nabla \xi+\alpha \nabla u_{t} \cdot \nabla \xi\right] \mathrm{d} \mathbf{x} \mathrm{d} t \\
& =\int_{\Omega} v_{0} \cdot \xi(0, \cdot) \mathrm{d} \mathbf{x}-\int_{\Omega_{T}} f(\mathbf{x}, u) \xi \mathrm{d} \mathbf{x} \mathrm{d} t
\end{aligned}
$$

(iv) for almost every $t \in[0, T]$,

$$
E_{p}\left[u(t, \cdot), u_{t}(t, \cdot)\right]-E_{p}\left[u_{0}, v_{0}\right] \leq-\alpha \int_{0}^{t} \int_{\Omega}\left|\nabla u_{t}\right|^{2} \mathrm{~d} \mathbf{x} \mathrm{d} t .
$$

2.3. Discretization in time and space. Let $\mathcal{T}_{h}$ be a quasi-uniform triangulation of a polygonal or polyhedral domain $\Omega \subset \mathbb{R}^{d}, d=2,3$ into triangles or tetrahedra of maximal diameter $h>0$, i.e., $\bar{\Omega}=\bigcup_{K \in \mathcal{T}_{h}} \bar{K}$. Let $\mathcal{N}_{h}=\left\{\mathbf{x}_{\ell}\right\}_{\ell \in L}$ denote the set of all nodes of $\mathcal{T}_{h}$. Let $V_{h}=\left\{U \in C(\bar{\Omega}): U\right.$ is affine on $\left.K, \forall K \in \mathcal{T}_{h}\right\}$ be the finite element space, and recall the nodal interpolation operator $\mathcal{I}_{h}: C(\bar{\Omega}) \rightarrow V_{h}$, such that $\mathcal{I}_{h} \psi=\sum_{\mathbf{z} \in \mathcal{N}_{h}} \psi(\mathbf{z}) \varphi_{\mathbf{z}} ;$ here, $\left\{\varphi_{\mathbf{z}}: \mathbf{z} \in \mathcal{N}_{h}\right\} \subset V_{h}$ denotes the nodal basis for $V_{h}$, and $\psi \in C(\bar{\Omega})$. Moreover, we use the $L^{2}(\Omega)$-orthogonal projection $P_{h}: L^{2}(\Omega) \rightarrow V_{h}$; i.e., for every $\varphi \in L^{2}(\Omega)$ there exists a unique $P_{h} \varphi \in V_{h}$, satisfying $\left(\varphi-P_{h} \varphi, \Psi\right)=0$ for all $\Psi \in V_{h}$.

Given a time-step size $k>0$, and a sequence $\left\{\varphi^{j}\right\}$ in some Banach space $X$, we set $d_{t} \varphi^{j}:=k^{-1}\left\{\varphi^{j}-\varphi^{j-1}\right\}$ for $j \geq 1$, and $d_{t}^{2} \varphi^{j} \equiv d_{t}\left(d_{t} \varphi^{j}\right)=k^{-2}\left\{\varphi^{j}-2 \varphi^{j-1}+\right.$ $\left.\varphi^{j-2}\right\}$ for $j \geq 2$. Note that $\left(d_{t} \varphi^{j}, \varphi^{j}\right)=\frac{1}{2} d_{t}\left\|\varphi^{j}\right\|^{2}+\frac{k}{2}\left\|d_{t} \varphi^{j}\right\|^{2}$ if $X$ is a Hilbert space. Piecewise constant interpolations of $\left\{\varphi^{j}\right\}$ are defined for $t \in\left[t_{j}, t_{j+1}\right)$, and $0 \leq j \leq J-1$ by

$$
\varphi^{-}(t):=\varphi^{j} \quad \text { and } \quad \varphi^{+}(t):=\varphi^{j+1}
$$


and a piecewise affine interpolation on $\left[t_{j}, t_{j+1}\right)$ is defined by

$$
\varphi(t):=\frac{t-t_{j}}{k} \varphi^{j+1}+\frac{t_{j+1}-t}{k} \varphi^{j} .
$$

Then $\left\|\phi^{ \pm}-\phi\right\|_{X} \leq 2 k\left\|d_{t} \phi\right\|_{X}$.

\section{An implicit Finite ELEMENT DisCRETIZATION OF (1.2)}

In the following, let $\left(U^{0}, V^{0}\right) \equiv\left(P_{L^{2}} u_{0}, P_{L^{2}} v_{0}\right) \in\left[V_{h}\right]^{2}$, and $U^{-1}:=U^{0}-k V^{0}$. The main goal in this section is to show that iterates of Scheme A below exist, satisfy a discrete energy law, and converge to weak solutions of (1.2) in the sense of Definition 2.1, for discretization, regularization, and perturbation parameters tending to zero.

Scheme A. Let $U^{-1}, U^{0} \in V_{h}$ be given. For every $1 \leq j \leq J$, find $U^{j} \in V_{h}$ such that for all $W \in V_{h}$,

$$
\begin{aligned}
\left(d_{t}^{2} U^{j}, W\right)+\left(\left[\left|\nabla U^{j}\right|^{2}+\delta(\mathbf{x})\right]\right. & \left.\frac{p_{\epsilon}(\mathbf{x})-2}{2} \nabla U^{j}, \nabla W\right) \\
& +\alpha\left(\nabla d_{t} U^{j}, \nabla W\right)+\left(\tilde{f}\left(\mathbf{x}, U^{j}, U^{j-1}\right), W\right)=0,
\end{aligned}
$$

for some $\delta \in L^{\infty}(\Omega,[0,1])$, a function $p_{\epsilon} \in C(\bar{\Omega},(1, \infty))$ approximating $p$, and

$$
\tilde{f}(\mathbf{x}, a, b)=\left\{\begin{aligned}
\frac{F(\mathbf{x}, a)-F(\mathbf{x}, b)}{a-b} & \text { if } a \neq b, \\
f(\mathbf{x}, a) & \text { if } a=b .
\end{aligned}\right.
$$

The latter construction is to validate a discrete energy inequality for iterates $\left(U^{j}, V^{j}\right):=\left(U^{j}, d_{t} U^{j}\right)(1 \leq j \leq J)$ solving (3.1), where $\left(\varphi, \psi \in V_{h}\right)$

$$
E_{p_{\epsilon}}^{\delta}[\varphi, \psi]:=\int_{\Omega}\left[\frac{1}{p_{\epsilon}(\mathbf{x})}\left[|\nabla \varphi|^{2}+\delta(\mathbf{x})\right]^{\frac{p_{\epsilon}(\mathbf{x})}{2}}+\frac{1}{2}|\psi|^{2}+F(\mathbf{x}, \varphi)\right] \mathrm{d} \mathbf{x} .
$$

In a first step, we show the well-posedness of Scheme $\mathrm{A}$ and the convergence of iterates to weak solutions of (1.2) for $\left(k, h, \delta, p_{\epsilon}-p\right) \rightarrow 0$ for finite initial energies, provided that $1<\gamma<p_{-}$, for $p_{-} \geq 2$.

Theorem 3.1. Let $T>0, \Omega \subset \mathbb{R}^{d}, d=2,3$ be a bounded Lipschitz domain, $\alpha \geq 0$, and $p \in C(\bar{\Omega},(1, \infty))$ such that (1.1) holds. Assume that $U^{0} \rightarrow u_{0}$ in $W_{0}^{1, p(\mathbf{x})}(\Omega)$, and $V^{0} \rightarrow v_{0}$ in $L^{2}(\Omega)$ for $h \rightarrow 0$, and let $f: \Omega \times \mathbb{R} \rightarrow \mathbb{R}$ be a continuous function which satisfies (2.3), with

$$
1<\gamma<p_{-} .
$$

(i) For sufficiently small $k, h>0, \delta \in L^{\infty}(\Omega,[0,1])$, and given $p_{\epsilon} \in C(\bar{\Omega},(1, \infty))$, which satisfies (1.1) for every $\epsilon \geq 0$, and

$$
p_{\epsilon}(\mathbf{x}) \geq p(\mathbf{x}) \quad \text { in } \Omega, \quad \text { such that }\left\|p-p_{\epsilon}\right\|_{L^{\infty}(\Omega)} \leq \epsilon,
$$

there exists $\left\{U^{j}\right\}_{j=1}^{J} \subset V_{h}$ which solves (3.1) and satisfies the discrete energy inequality

$$
E_{p_{\epsilon}}^{\delta}\left[U^{j}, V^{j}\right]+\frac{k^{2}}{2} \sum_{\ell=1}^{j}\left\|d_{t}^{2} U^{\ell}\right\|_{L^{2}}^{2} \leq E_{p_{\epsilon}}^{\delta}\left[U^{0}, V^{0}\right] \quad(1 \leq j \leq J) .
$$


(ii) Let $\alpha>0$ and $p_{-} \geq 2$. For every $t \in\left[t_{j-1}, t_{j}\right)(1 \leq j \leq J)$, and

$$
U(t, \cdot) \equiv U_{k, h, \delta(\mathbf{x}), \epsilon}(t, \cdot):=\frac{t-t_{j-1}}{k} U^{j}+\frac{t_{j}-t}{k} U^{j-1} \quad \text { in } \Omega,
$$

there exist a convergent subsequence $\{U\}$, and $u \in L^{\infty}\left(0, T ; W_{0}^{1, p(\mathbf{x})}(\Omega)\right)$, such that

$$
U \stackrel{*}{\rightarrow} u \quad \text { in } L^{\infty}\left(0, T ; W^{1, p(\mathbf{x})}(\Omega)\right) \quad(k, h, \delta(\mathbf{x}), \epsilon) \rightarrow 0 .
$$

Moreover, $u: \Omega_{T} \rightarrow \mathbb{R}$ is a weak solution of (1.2) in the sense of Definition 2.1.

Remark 1. Let $f(\mathbf{x}, u)=-|u|^{\gamma-2} u$, and $p \geq 2$ be constant in (1.2). (i) Assume $\alpha=0$. If $\gamma>p$, solutions with negative initial energy blow up in finite time [4, 20].

(ii) The same behavior is known for $\alpha>0$; see [31, 25].

The proof is split into two parts, where the first addresses existence of solutions $\left\{U^{j}\right\}_{1 \leq j \leq J} \subset V_{h}$ of Scheme $\mathrm{A}$, as well as the discrete energy inequality (3.5); the second part verifies convergence of iterates towards weak solutions for $(k, h, \delta(\mathbf{x}), \epsilon) \rightarrow 0$.

Proof (Theorem 3.1, part (i)). Step 1. Existence of sequences $\left\{U^{j}\right\}_{j=1}^{J} \subset V_{h}$ that solve (3.1). For every $k, h>0$, and $p_{-}>1$, solutions $U^{j} \in V_{h}$ of Scheme A minimize the continuous functional $E_{k, h}^{j}: V_{h} \rightarrow \mathbb{R}$, with $(j \geq 1)$

$$
\begin{aligned}
E_{k, h}^{j}[W]:= & \int_{\Omega}\left[\frac{1}{2 k^{2}}\left|W-2 U^{j-1}+U^{j-2}\right|^{2}+\frac{1}{p_{\epsilon}(\mathbf{x})}\left(|\nabla W|^{2}+\delta(\mathbf{x})\right)^{\frac{p_{\epsilon}(\mathbf{x})}{2}}\right. \\
& \left.+\frac{\alpha}{2 k}\left|\nabla\left(W-U^{j-1}\right)\right|^{2}+\frac{1}{\theta} F\left(\mathbf{x}, \theta W+(1-\theta) U^{j-1}\right)\right] \mathrm{d} \mathbf{x},
\end{aligned}
$$

for some $\theta \in(0,1)$. Here, we use the fact that $\tilde{f}(\mathbf{x}, a, b)=f(\mathbf{x}, \theta a+(1-\theta) b)$ for some $\theta \in(0,1)$. In order to bound the last term in (3.6), we recall (2.3) and use Poincaré's and Young's inequalities to find

$$
\begin{aligned}
\int_{\Omega}|F(\mathbf{x}, W)| \mathrm{d} \mathbf{x} & =\int_{\Omega}\left|\int_{0}^{W} f(\mathbf{x}, s) \mathrm{d} s\right| \mathrm{d} \mathbf{x} \\
& \leq \int_{\Omega} \int_{0}^{W}\left[C_{1}|s|^{\gamma-1}+h_{f}(\mathbf{x})\right] \mathrm{d} s \mathrm{~d} \mathbf{x} \\
& \leq \frac{C_{1}}{\gamma}\|W\|_{L^{\gamma}}^{\gamma}+\left\|h_{f}\right\|_{L^{\infty}}\|W\|_{L^{1}} \\
& \leq C\left[\frac{C_{1}}{\gamma}+1\right]\|\nabla W\|_{L^{\gamma}}^{\gamma}+\left\|h_{f}\right\|_{L^{\infty}}^{\frac{\gamma}{\gamma-1}} .
\end{aligned}
$$

Since $\left[p_{\epsilon}\right]_{-}>\gamma$, there exists a constant $\bar{C}=\bar{C}\left(h_{f}\right)>0$, such that

$$
\int_{\Omega}|F(\mathbf{x}, W)| \mathrm{d} \mathbf{x} \leq \bar{C}+\int_{\Omega} \frac{1}{2 p_{\epsilon}(\mathbf{x})}\left(|\nabla W|^{2}+\delta(\mathbf{x})\right)^{\frac{p_{\epsilon}(\mathbf{x})}{2}} \mathrm{~d} \mathbf{x} .
$$

Hence, we may conclude coercivity of $E_{k, h}^{j}: V_{h} \rightarrow \mathbb{R}$, and hence existence of a (unique) minimizer $U^{j} \in V_{h}$.

Step 2. Discrete energy estimate (3.5). Choose $W=d_{t} U^{j}$ in (3.1). Then a convexity argument and summation over all $1 \leq \ell \leq j$ lead to the discrete energy 
inequality

$$
\begin{aligned}
E_{p_{\epsilon}}^{\delta}\left[U^{j}, V^{j}\right]+\frac{k^{2}}{2} \sum_{\ell=1}^{j}\left\|d_{t} V^{j}\right\|_{L^{2}}^{2} & \\
& +\alpha \sum_{\ell=1}^{j}\left\|\nabla V^{j}\right\|_{L^{2}}^{2} \leq E_{p_{\epsilon}}^{\delta}\left[U^{0}, V^{0}\right] \quad(1 \leq j \leq J) .
\end{aligned}
$$

Since $F\left(\mathbf{x}, U^{j}\right)=\int_{0}^{U^{j}} f(\mathbf{x}, s) \mathrm{d} s$, by the growth condition (2.3), and Sobolev embedding, we obtain

$$
\int_{\Omega}\left|F\left(\mathbf{x}, U^{j}\right)\right| \mathrm{d} \mathbf{x} \leq\left\|h_{f}\right\|_{L^{\frac{\gamma}{\gamma-1}}}\left\|U^{j}\right\|_{L^{\gamma}}+C_{1}\left\|U^{j}\right\|_{L^{\gamma}}^{\gamma}
$$

Since $\left[p_{\epsilon}\right]_{-}>\gamma$, we may proceed as in (3.7) and obtain from (3.8)

$$
\begin{aligned}
& \frac{1}{2} \int_{\Omega}\left[\frac{1}{p_{\epsilon}(\mathbf{x})}\left[\left|\nabla U^{j}\right|^{2}+\delta(\mathbf{x})\right]^{\frac{p_{\epsilon}(\mathbf{x})}{2}}+\left|V^{j}\right|^{2}\right] \mathrm{d} \mathbf{x} \\
& \quad+\frac{k}{2} \sum_{\ell=1}^{j} \int_{\Omega}\left[k\left|d_{t} V^{\ell}\right|^{2}+\alpha\left|\nabla V^{\ell}\right|^{2}\right] \mathrm{d} \mathbf{x} \leq E_{p_{\epsilon}}^{\delta}\left[U^{0}, V^{0}\right]+\bar{C} .
\end{aligned}
$$

We use $V^{j}=d_{t} U^{j} \in V_{h}$ to restate (3.1) in the following way:

$$
\begin{aligned}
\left(d_{t} V^{j}, W\right) & +\left(\left[\left|\nabla U^{j}\right|^{2}+\delta(\mathbf{x})\right]^{\frac{p_{\epsilon}(\mathbf{x})-2}{2}} \nabla U^{j}, \nabla W\right) \\
& +\alpha\left(\nabla d_{t} U^{j}, \nabla W\right)+\left(\tilde{f}\left(\mathbf{x}, U^{j}, U^{j-1}\right), W\right)=0, \\
\left(V^{j}, \Psi\right)- & \left(d_{t} U^{j}, \Psi\right)=0,
\end{aligned}
$$

for all $(W, \Psi) \in\left[V_{h}\right]^{2}$. This formulation of the problem leads to the following result.

Lemma 3.1. Let $\left\{U^{j}\right\}_{j \geq-1} \subset V_{h}$ solve Scheme A. For all $T>0$, and $\Psi \in$ $W^{1,1}\left(0, T ; V_{h}\right) \cap L^{2}\left(0, T ; V_{h}\right)$, it follows that

$$
\begin{aligned}
\mid \int_{0}^{T} & {\left[-\left(U_{t}, \Psi_{t}\right)+\left(\left[|\nabla U|^{2}+\delta(\mathbf{x})\right]^{\frac{p_{\epsilon}(\mathbf{x})-2}{2}} \nabla U, \nabla \Psi\right)\right.} \\
& \left.+\alpha\left(\nabla U_{t}, \nabla \Psi\right)+(f(\mathbf{x}, U), \Psi)\right] \mathrm{d} s+(V(T, \cdot), \Psi(T, \cdot))-(V(0, \cdot), \Psi(0, \cdot)) \mid \\
\leq & \left|\int_{0}^{T}\left(\left[|\nabla U|^{2}+\delta(\mathbf{x})\right]^{\frac{p_{\epsilon}(\mathbf{x})-2}{2}} \nabla U-\left[\left|\nabla U^{+}\right|^{2}+\delta(\mathbf{x})\right]^{\frac{p_{\epsilon}(\mathbf{x})-2}{2}} \nabla U^{+}, \nabla \Psi\right) \mathrm{d} s\right| \\
& +\left|\int_{0}^{T}\left(V-V^{+}, \Psi_{t}\right) \mathrm{d} s\right|+\left|\int_{0}^{T}\left(f(\mathbf{x}, U)-\tilde{f}\left(\mathbf{x}, U^{+}, U^{-}\right), \Psi\right) \mathrm{d} s\right| .
\end{aligned}
$$


Proof. We rewrite (3.10) as follows: for all $\Psi, \Phi \in W^{1,1}\left(0, T ; V_{h}\right) \cap L^{2}\left(0, T ; V_{h}\right)$,

$$
\begin{aligned}
& \int_{0}^{T}\left[\left(V_{t}, \Psi\right)+\left(\left[\left|\nabla U^{+}\right|^{2}+\delta(\mathbf{x})\right]^{\frac{p_{\epsilon}(\mathbf{x})-2}{2}} \nabla U^{+}, \nabla \Psi\right)\right. \\
& \left.+\alpha\left(\nabla U_{t}, \nabla \Psi\right)+\left(\tilde{f}\left(\mathbf{x}, U^{+}, U^{-}\right), \Psi\right)\right] \mathrm{d} t=0, \\
& \int_{0}^{T}\left[\left(V^{+}, \Psi\right)-\left(U_{t}, \Psi\right)\right] \mathrm{d} t=0 .
\end{aligned}
$$

Integration by parts in time in the first term in (3.13) yields

$$
\begin{aligned}
\int_{0}^{T}\left(V_{t}, \Psi\right) \mathrm{d} t= & -\int_{0}^{T}\left(V, \Psi_{t}\right) \mathrm{d} t+(V(T, \cdot), \Psi(T, \cdot))-(V(0, \cdot), \Psi(0, \cdot)) \\
= & -\int_{0}^{T}\left(U_{t}, \Psi_{t}\right) \mathrm{d} t-\int_{0}^{T}\left(V^{+}-V, \Psi_{t}\right) \mathrm{d} t \\
& +(V(T, \cdot), \Psi(T, \cdot))-(V(0, \cdot), \Psi(0, \cdot)),
\end{aligned}
$$

thanks to (3.14). Putting things together verifies the assertion of the lemma.

The following result gives uniform bounds for $\{V\}$.

Lemma 3.2. Suppose that the assumptions of Theorem 3.1 (ii) are valid. Then

$$
\left\|V_{t}\right\|_{L^{2}\left(0, T ; W^{-1,\left[p_{-}\right]^{\prime}}\right)}+\|V\|_{L^{2}\left(0, T ; W^{1,2}\right)} \leq C .
$$

Proof. We use (3.13), and employ $W^{1, p}$-stability of $P_{L^{2}}$, see [9, Theorems $3 \& 4$ ], to conclude

$$
\begin{aligned}
\left\|V_{t}(s, \cdot)\right\|_{W^{-1,\left[p_{-}\right]^{\prime}}} & :=\sup _{\varphi \in W_{0}^{1, p_{-}}} \frac{\left(V_{t}(s, \cdot), P_{L^{2}} \varphi\right)}{\|\varphi\|_{W^{1, p_{-}}}} \\
& \leq C\left[\left\|\nabla U^{+}(s, \cdot)\right\|_{L^{p_{-}}}^{p_{-}}+\left\|\nabla U_{t}(s, \cdot)\right\|_{L^{2}}+\|U(s, \cdot)\|_{L^{p_{-}}}^{p_{-}-1}+1\right] .
\end{aligned}
$$

Estimate (3.9) then implies the first part of the assertion. The second part follows from (3.9) and

$$
\|V\|_{L^{2}\left(0, T ; W^{1,2}\right)} \leq\left\|V^{-}\right\|_{L^{2}\left(0, T ; W^{1,2}\right)}+\left\|V^{+}\right\|_{L^{2}\left(0, T ; W^{1,2}\right)} .
$$

Proof (Theorem 3.1. part (ii)). Step 3. Passing to the limit $(k, h, \delta(\mathbf{x}), \epsilon) \rightarrow 0$ in (3.1). It follows from (3.9) that there exist

$$
u \in L^{\infty}\left(0, T ; W_{0}^{1, p(\mathbf{x})}(\Omega)\right) \cap W^{1, \infty}\left(0, T ; L^{2}(\Omega)\right) \cap W^{1,2}\left(0, T ; W_{0}^{1,2}(\Omega)\right),
$$

and a convergent subsequence of $\{U\}_{k, h, \delta(\mathbf{x}), \epsilon}$ such that for $(k, h, \delta(\mathbf{x}), \epsilon) \rightarrow 0$,

$$
\begin{aligned}
U \stackrel{*}{*} u & \text { in } L^{\infty}\left(0, T ; W_{0}^{1, p(\mathbf{x})}(\Omega)\right) \cap W^{1, \infty}\left(0, T ; L^{2}(\Omega)\right), \\
U \rightarrow u & \text { in } W^{1,2}\left(0, T ; W_{0}^{1,2}(\Omega)\right), \\
{\left[\left|\nabla U^{+}\right|^{2}+\delta\right]^{\frac{p_{-}-2}{2}} \nabla U^{+} \stackrel{*}{\rightarrow} b } & \text { in } L^{\infty}\left(0, T ; L^{\frac{p(\mathbf{x})}{p(\mathbf{x})-1}}(\Omega)\right), \\
V \rightarrow u_{t} & \text { in } W^{1,2}\left(0, T ; W^{-1,\left[p_{-}\right]^{\prime}}(\Omega)\right) \cap L^{2}\left(0, T ; W_{0}^{1,2}(\Omega)\right), \\
U, U^{+}, U^{-} \rightarrow u & \text { in } W^{1,2}\left(0, T ; L^{2}(\Omega)\right), \\
f(\cdot, U), \tilde{f}\left(\cdot, U^{+}, U^{-}\right) \rightarrow f(\cdot, u) & \text { in } L^{\frac{\gamma}{\gamma-1}}\left(\Omega_{T}\right),
\end{aligned}
$$


thanks to (3.5) and Lemma 3.2, property $(3.15)_{5}$ is a consequence of the control

$$
\left\|V^{+}-V\right\|_{L^{2}\left(\Omega_{T}\right)} \leq \sqrt{k}\left[\sqrt{k}\left\|V_{t}\right\|_{L^{2}\left(\Omega_{T}\right)}\right] \leq \sqrt{k} E_{p_{\epsilon}}^{\delta}\left[U_{0}, V_{0}\right]
$$

and follows from the Aubin-Lions compactness result. Property 3.15$)_{6}$ is a consequence of the following bound, which is uniform in $(k, h, \delta(\mathbf{x}), \epsilon)$ :

$$
\int_{\Omega_{T}}|f(\cdot, U)|^{\frac{\gamma}{\gamma-1}} \mathrm{~d} \mathbf{x} \mathrm{d} t \leq C,
$$

thanks to $\gamma<p_{-}$, and (3.15) 5 ; the second property here uses $\tilde{f}\left(\cdot, U^{+}, U^{-}\right)=$ $f\left(\cdot, \theta U^{+}+(1-\theta) U^{-}\right)$for some $\theta \in(0,1)$.

We can now identify limits in (3.12): let $\Psi=\mathcal{I}_{h} \xi(t, \cdot)$ for $\xi \in C_{0}^{\infty}([0, T) \times \Omega)$. Thanks to $\left[\operatorname{Id}-\mathcal{I}_{h}\right] \xi(t, \cdot) \rightarrow 0$ in $C_{0}^{\infty}(\Omega)(h \rightarrow 0)$ for every $t \in[0, T]$, and (3.15), (3.16), the right-hand side of (3.12) vanishes for $(k, h, \delta(\mathbf{x}), \epsilon) \rightarrow 0$, and we obtain

$$
\begin{array}{r}
\int_{0}^{T}\left[-\left(u_{t}, \xi_{t}\right)+(b, \nabla \xi)+\alpha\left(\nabla u_{t}, \nabla \xi\right)+(f(\mathbf{x}, u), \xi)\right] \mathrm{d} s=\left(v_{0}, \xi(0, \cdot)\right) \\
\forall \xi \in C_{0}^{\infty}([0, T) \times \Omega) .
\end{array}
$$

It remains to show that $b=|\nabla u|^{p(\mathbf{x})-2} \nabla u$. For this purpose, monotonicity of $-\Delta_{p(\mathbf{x})}: W_{0}^{1, p(\mathbf{x})}(\Omega) \rightarrow\left[W_{0}^{1, p(\mathbf{x})}(\Omega)\right]^{*}$ implies

$$
\left(\left[\left|\nabla U^{+}\right|^{2}+\delta\right]^{\frac{p_{\epsilon}(\mathbf{x})-2}{2}} \nabla U^{+}-\left[\left|\nabla \mathcal{I}_{h} \xi^{+}\right|^{2}+\delta\right]^{\frac{p_{\epsilon}(\mathbf{x})-2}{2}} \nabla \mathcal{I}_{h} \xi^{+}, \nabla\left[U^{+}-\mathcal{I}_{h} \xi^{+}\right]\right) \geq 0,
$$

for every $W(t, \cdot)=\left[U^{+}-\mathcal{I}_{h} \xi^{+}\right](t, \cdot) \in V_{h}$, where $\xi \in C_{0}^{\infty}([0, T) \times \Omega)$. We use equation (3.13) to conclude from this inequality that

$$
\begin{aligned}
\int_{0}^{T}[ & -\left(\tilde{f}\left(\cdot, U^{+}, U^{-}\right), U^{+}-\mathcal{I}_{h} \xi^{+}\right)-\left(V_{t}, U^{+}-\mathcal{I}_{h} \xi^{+}\right)-\alpha\left(\nabla U_{t}, \nabla\left[U^{+}-\mathcal{I}_{h} \xi^{+}\right]\right) \\
& \left.-\left(\left[\left|\nabla \mathcal{I}_{h} \xi^{+}\right|^{2}+\delta\right]^{\frac{p_{\epsilon}(\mathbf{x})-2}{2}} \nabla \mathcal{I}_{h} \xi^{+}, \nabla\left[U^{+}-\mathcal{I}_{h} \xi^{+}\right]\right)\right] \mathrm{d} t \geq 0 .
\end{aligned}
$$

Passing to the limit $(k, h, \delta(\mathbf{x}), \epsilon) \rightarrow 0$, and again using (3.15), (3.17), together with $\left[\operatorname{Id}-\mathcal{I}_{h}\right] \xi(t, \cdot) \rightarrow 0(h \rightarrow 0)$ in $W^{1, \infty}(\Omega)$ then yields

$$
\int_{0}^{T}\left(b-|\nabla \xi|^{p(\mathbf{x})-2} \nabla \xi, \nabla[u-\xi]\right) \mathrm{d} t \geq 0 \quad \forall \xi \in C_{0}^{\infty}([0, T) \times \Omega) .
$$

Here, we use the following property, which employs $W^{1, \infty}(\Omega)$-stability of the Lagrange interpolation operator,

$$
\begin{aligned}
& \left\|\left|\nabla \mathcal{I}_{h} \xi^{+}\right|^{p(\mathbf{x})-2}\left(1-\left|\nabla \mathcal{I}_{h} \xi^{+}\right|^{p_{\epsilon}(\mathbf{x})-p(\mathbf{x})}\right) \nabla \mathcal{I}_{h} \xi^{+}\right\|_{L^{1}} \\
& \leq C\left(1+\left\|\nabla \xi^{+}\right\|_{L^{\infty}}\right)^{p_{+}-1}\left\|1-\left|\nabla \mathcal{I}_{h} \xi^{+}\right|^{p_{\epsilon}(\mathbf{x})-p(\mathbf{x})}\right\|_{L^{1}} .
\end{aligned}
$$

We split the second factor as follows:

$$
\left.\left[\int_{\left\{\left|\nabla \mathcal{I}_{h} \xi^{+}\right| \leq 2\right\}}+\int_{\left\{\left|\nabla \mathcal{I}_{h} \xi^{+}\right|>2\right\}}\right]|1-| \nabla \mathcal{I}_{h} \xi^{+}\right|^{p_{\epsilon}(\mathbf{x})-p(\mathbf{x}) \mid} \mid \mathrm{d} \mathbf{x} .
$$

Convergence to zero of the first term for $\epsilon \rightarrow 0$ is immediate; for the second term, we calculate that

$$
\int_{\left\{\left|\nabla \mathcal{I}_{h} \xi^{+}\right|>2\right\}}\left|1-\exp \left(\epsilon \ln \left[\left|\nabla \mathcal{I}_{h} \xi^{+}\right|\right]\right)\right| \mathrm{d} \mathbf{x} \rightarrow 0 \quad(\epsilon \rightarrow 0) .
$$


Let us come back to (3.18): Choosing $\xi=u \pm \alpha \zeta$, and letting $\alpha \rightarrow 0$, we employ monotonicity of $\Delta_{p(\mathbf{x})}: W_{0}^{1, p(\mathbf{x})}(\Omega) \rightarrow\left[W_{0}^{1, p(\mathbf{x})}(\Omega)\right]^{*}$ to conclude that

$$
\int_{0}^{T}\left(b-|\nabla u|^{p(\mathbf{x})-2} \nabla u, \nabla \zeta\right) \mathrm{d} t=0 \quad \forall \zeta \in C_{0}^{\infty}([0, T) \times \Omega),
$$

which validates $b=|\nabla u|^{p(\mathbf{x})-2} \nabla u$. This identification in (3.17) verifies property (iii) of Definition 2.1

Properties (ii), resp. (iii), of Definition 2.1 are now immediate consequences of (i), resp. (3.5).

Remark 2. 1. Suppose that $F: \Omega \times \mathbb{R} \rightarrow \mathbb{R}$ is convex in the second argument. Then we can use the following discretization instead of (3.1):

$$
\begin{aligned}
& \left(d_{t}^{2} U^{j}, W\right)+\left(\left[\left|\nabla U^{j}\right|^{2}+\delta(\mathbf{x})\right]^{\frac{p_{\epsilon}(\mathbf{x})-2}{2}} \nabla U^{j}, \nabla W\right) \\
& +\alpha\left(\nabla d_{t} U^{j}, \nabla W\right)+\left(f\left(\mathbf{x}, U^{j}\right), W\right)=0,
\end{aligned}
$$

for all $W \in V_{h}$. In this case, existing solutions $\left\{U^{j}\right\}_{j>0} \subset V_{h}$ minimize (3.6), with $\theta=1$, and satisfy (3.5) as well.

2. For general continuous $f: \Omega \times \mathbb{R} \rightarrow \mathbb{R}$, we may employ scheme (3.19) as well, or the following semi-implicit variant,

$$
\begin{aligned}
\left(d_{t}^{2} U^{j}, W\right)+\left(\left[\left|\nabla U^{j}\right|^{2}+\delta(\mathbf{x})\right]^{\frac{p_{\epsilon}(\mathbf{x})-2}{2}} \nabla\right. & \left.U^{j}, \nabla W\right) \\
& +\alpha\left(\nabla d_{t} U^{j}, \nabla W\right)+\left(f\left(\mathbf{x}, U^{j-1}\right), W\right)=0,
\end{aligned}
$$

for all $W \in V_{h}$. However, in both cases, (3.3) has to be sharpened to $\gamma \leq \frac{p_{-}}{2}+1$, and the discrete energy inequality (3.5) does not hold any more. The reason for this comes from the following modifications of $(3.8)(1 \leq j \leq J)$ :

$$
\begin{aligned}
\max _{1 \leq \ell \leq j} & {\left[\frac{1}{2}\left\|V^{\ell}\right\|_{L^{2}}^{2}+\int_{\Omega} \frac{1}{p_{\epsilon}(\mathbf{x})}\left(\left|\nabla U^{\ell}\right|^{2}+\delta(\mathbf{x})\right)^{\frac{p_{\epsilon}(\mathbf{x})}{2}} \mathrm{~d} \mathbf{x}\right] } \\
& +\frac{k^{2}}{2} \sum_{\ell=1}^{j}\left\|d_{t} V^{j}\right\|_{L^{2}}^{2}+\alpha k \sum_{\ell=1}^{j}\left\|\nabla V^{j}\right\|_{L^{2}}^{2} \\
\leq & \frac{1}{2}\left\|V^{0}\right\|_{L^{2}}^{2}+\int_{\Omega} \frac{1}{p_{\epsilon}(\mathbf{x})}\left(\left|\nabla U^{0}\right|^{2}+\delta(\mathbf{x})\right)^{\frac{p_{\epsilon}(\mathbf{x})}{2}} \mathrm{~d} \mathbf{x}+k \sum_{\ell=1}^{j}\left|\left(f\left(\mathbf{x}, U^{\ell}\right), V^{\ell}\right)\right| .
\end{aligned}
$$

We use Young's inequality and (2.3) to bound the last term as follows:

$$
\leq k \sum_{\ell=1}^{j}\left[\mid f\left(\mathbf{x}, U^{\ell}\right)\left\|_{L^{[2 *]^{\prime}}}\right\| V^{\ell} \|_{L^{2 *}}\right] \leq k \sum_{\ell=1}^{j}\left[C \alpha\left\|U^{\ell}\right\|_{L^{[2 *]^{\prime}(\gamma-1)}}^{2(\gamma-1)}+\frac{\alpha}{2}\left\|\nabla V^{\ell}\right\|_{L^{2}}^{2}\right],
$$

where we put $h_{f} \equiv 1$ in (2.3) for simplicity. Hence, in order to use the discrete version of Gronwall's lemma, we need $2(\gamma-1) \leq p_{-}$.

\section{A simple fixed point scheme to solve Algorithm A}

For every $j \geq 1$ in Scheme A, a nonlinear algebraic equation has to be solved; a simple fixed point strategy, together with a stopping criterion, could be as follows. 
Algorithm $\mathbf{A}_{0}$. Let $U^{0} \in V_{h}, V^{0}:=d_{t} U^{0} \in V_{h}$, and set $j:=1$.

1. Set $U^{j, 0}:=U^{j-1}$ and $\ell=0$.

2. Compute $U^{j, \ell} \in V_{h}$ such that for all $W \in V_{h}$,

$$
\begin{aligned}
& \left(\frac{U^{j, \ell}-U^{j-1}}{k^{2}}, W\right)+\left(\left[\left|\nabla U^{j, \ell-1}\right|^{2}+\delta(\mathbf{x})\right]^{\frac{p_{\epsilon}(\mathbf{x})-2}{2}} \nabla U^{j, \ell}, \nabla W\right) \\
& \quad+\frac{\alpha}{k}\left(\nabla\left[U^{j, \ell}-U^{j-1}\right], \nabla W\right)+\left(\tilde{f}\left(\mathbf{x}, U^{j, \ell-1}, U^{j-1}\right), W\right) \\
& =\frac{1}{k}\left(d_{t} U^{j-1}, W\right) .
\end{aligned}
$$

3. For fixed $\theta>0$, stop if

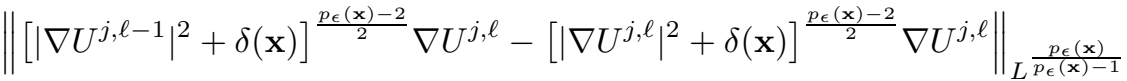

$$
\begin{aligned}
& +\left\|\tilde{f}\left(\mathbf{x}, U^{j, \ell}, U^{j-1}\right)-\tilde{f}\left(\mathbf{x}, U^{j, \ell-1}, U^{j-1}\right)\right\|_{L^{2}} \leq \theta,
\end{aligned}
$$

set $U^{j}:=U^{j, \ell}, j:=j+1$, and go to Step 1 .

4. Set $\ell:=\ell+1$, and go to Step 2.

Unfortunately, it is not clear whether a contraction property holds, the reason being the modified nonlinearity $\tilde{f}$. For this reason, we base the following algorithm on discretization (3.19); see Remark 2, which discusses existence of solutions for monotone $f$, or more general situations, where (2.3) holds for $\gamma \leq \frac{p_{-}}{2}+1$, and thus allows for convergence of iterates towards weak solutions of (1.2) in the sense of Definition 2.1.

Algorithm $\mathbf{A}_{1}$. Let $U^{0} \in V_{h}, V^{0}:=d_{t} U^{0} \in V_{h}$, and set $j:=1$.

1. Set $U^{j, 0}:=U^{j-1}$ and $\ell=0$.

2. Compute $U^{j, \ell} \in V_{h}$ such that for all $W \in V_{h}$,

$$
\begin{aligned}
& \left(\frac{U^{j, \ell}-U^{j-1}}{k^{2}}, W\right)+\left(\left[\left|\nabla U^{j, \ell-1}\right|^{2}+\delta(\mathbf{x})\right]^{\frac{p_{\epsilon}(\mathbf{x})-2}{2}} \nabla U^{j, \ell}, \nabla W\right) \\
& +\frac{\alpha}{k}\left(\nabla\left[U^{j, \ell}-U^{j-1}\right], \nabla W\right)+\left(f\left(\mathbf{x}, U^{j, \ell-1}\right), W\right)=\frac{1}{k}\left(d_{t} U^{j-1}, W\right)
\end{aligned}
$$

3. For fixed $\theta>0$, stop if

$$
\begin{aligned}
& \left\|\left[\left|\nabla U^{j, \ell-1}\right|^{2}+\delta(\mathbf{x})\right]^{\frac{p_{\epsilon}(\mathbf{x})-2}{2}} \nabla U^{j, \ell}-\left[\left|\nabla U^{j, \ell}\right|^{2}+\delta(\mathbf{x})\right]^{\frac{p_{\epsilon}(\mathbf{x})-2}{2}} \nabla U^{j, \ell}\right\|_{L} \frac{p_{\epsilon}(\mathbf{x})}{p_{\epsilon}(\mathbf{x})-1} \\
& +\left\|f\left(\mathbf{x}, U^{j, \ell}\right)-f\left(\mathbf{x}, U^{j, \ell-1}\right)\right\|_{L^{2}} \leq \theta,
\end{aligned}
$$

set $U^{j}:=U^{j, \ell}, j:=j+1$, and go to Step 1 .

4. Set $\ell:=\ell+1$, and go to Step 2.

Below, we validate overall convergence to weak solutions of (1.2) in the sense of Definition 2.1. For this purpose, we assume that $f$ is differentiable with respect to its second argument, and for some $1<r<\infty$,

$$
\left|\frac{\partial}{\partial s} f(\mathbf{x}, s)\right| \leq C\left(1+|s|^{r}\right) \quad \forall \mathbf{x} \in \Omega .
$$

Theorem 4.1. Suppose that the assumptions of Theorem 3.1 are valid, $\alpha>0$, and (4.5) holds. Let $\delta \in C(\bar{\Omega},[0,1])$ such that $\delta(\mathbf{x})>0$ if $p \equiv p(\mathbf{x})<3$, and $\delta(\mathbf{x})=0$ otherwise. For all $\ell \geq 0$, there exists a unique solution $U^{j, \ell} \in V_{h}$ to (4.3). 
Moreover, there exists $\tilde{C} \equiv \tilde{C}(d, r, \Omega)>0$ such that if for all $1 \leq j \leq J$ it follows that

$$
\begin{aligned}
& \alpha^{2} k^{2} h^{-d r}+\chi_{\left\{2 \leq p_{\epsilon}(\cdot) \leq 3\right\}}\left[\max _{\left\{2 \leq p_{\epsilon}(\cdot) \leq 3\right\}}[\delta(\cdot)]^{p_{\epsilon}(\cdot)-3} h^{-[d+2]}\right. \\
& \left.+\max _{\left\{2 \leq p_{\epsilon}(\cdot) \leq 3\right\}} h^{-[d+2]\left(p_{\epsilon}(\cdot)-2\right)}\right] \\
& +\chi_{\left\{p_{\epsilon}(\cdot)>3\right\}} h^{-[d+2]\left(p_{\epsilon}(\cdot)-2\right)}<\tilde{C}^{-1} k^{-2} \alpha^{2},
\end{aligned}
$$

then $(\ell \geq 1)$

$$
\begin{aligned}
& {\left[\left\|U^{j, \ell+1}-U^{j, \ell}\right\|_{L^{2}}^{2}+\alpha k\left\|\nabla\left[U^{j, \ell+1}-U^{j, \ell}\right]\right\|_{L^{2}}^{2}\right]} \\
& \quad \leq q\left[\left\|U^{j, \ell}-U^{j, \ell-1}\right\|_{L^{2}}^{2}+\alpha k\left\|\nabla\left[U^{j, \ell}-U^{j, \ell-1}\right]\right\|_{L^{2}}^{2}\right] \quad \text { where } q<1 .
\end{aligned}
$$

Finally, let either $F: \Omega \times \mathbb{R} \rightarrow \mathbb{R}$ be convex in the second argument, or else $\gamma \leq \frac{p_{-}}{2}+1$. Then iterates $\left\{U^{j, \bar{\ell}}\right\}_{j}$ of Algorithm $A_{1}$ which meet the stopping criterion in Step 3 subconverge to weak solutions of (1.2) in the sense of Definition 2.1, for $(k, h, \delta(\mathbf{x}), \epsilon, \theta) \rightarrow 0$, as is specified in Theorem 3.1 .

Remark 3. In Theorem 4.1 we assume $\alpha>0$; however, for $\alpha=0$, a corresponding convergence result can be derived for iterates $\left\{U^{j, \ell}\right\}_{\ell} \subset V_{h}$ of Algorithm $\mathrm{A}_{1}$ towards solutions $U^{j} \in V_{h}$ of Scheme A $(1 \leq j \leq J)$, provided a more restrictive mesh constraint than (4.6) holds.

Proof. Step 1. Contraction principle. Let $j \geq 1$ be fixed, and

$$
\rho_{p_{\epsilon}}\left(\nabla U^{j-1}\right)+\left\|d_{t} U^{j-1}\right\|_{L^{2}}^{2} \leq C,
$$

independently of $j$. For every $\ell \geq 1$, existence and uniqueness of a solution $U^{j, \ell} \in V_{h}$ follow from the Lax-Milgram theorem. To show convergence of $\left\{U^{j, \ell}\right\}_{\ell}$, we establish an $L^{2}(\Omega)$-contraction property (4.7). Let $E^{j, \ell}:=U^{j, \ell}-U^{j, \ell-1} \in V_{h}$; we subtract two successive versions of (4.3) and choose $W=E^{j, \ell}$ as a test function,

$$
\begin{aligned}
& \frac{1}{2}\left\|E^{j, \ell}\right\|_{L^{2}}^{2}+ \frac{\alpha k}{2}\left\|\nabla E^{j, \ell}\right\|_{L^{2}}^{2} \\
&+C \alpha^{-1} k^{3} \|\left[\left|\nabla U^{j, \ell-1}\right|^{2}+\delta(\mathbf{x})\right]^{\frac{p_{\epsilon}(\mathbf{x})-2}{2}} \nabla U^{j, \ell} \\
&-\left[\left|\nabla U^{j, \ell-2}\right|^{2}+\delta(\mathbf{x})\right]^{\frac{p_{\epsilon}(\mathbf{x})-2}{2}} \nabla U^{j, \ell-1} \|_{L^{2}}^{2}
\end{aligned}
$$

by Young's inequality.

The leading error term on the right-hand side of (4.9) is bounded by an inverse estimate, and (4.5), for some $\xi \in(0,1)$,

$$
\begin{aligned}
& \leq C k^{4}\left[1+\left\|\xi U^{j, \ell-1}+(1-\xi) U^{j, \ell-2}\right\|_{L^{\infty}}^{2 r}\right]\left\|E^{j, \ell-1}\right\|_{L^{2}}^{2} \\
& \leq C k^{4}\left[1+h^{-d r}\left\|\xi U^{j, \ell-1}+(1-\xi) U^{j, \ell-2}\right\|_{L^{2}}^{2 r}\right]\left\|E^{j, \ell-1}\right\|_{L^{2}}^{2} .
\end{aligned}
$$

Note that $\left\|U^{j, \ell}\right\|_{L^{2}} \leq C$, for all $\ell \geq 0$, which easily follows from choosing $W=U^{j, \ell}$ in (4.3), and using (4.8). 
Since $x \mapsto\left[|x|^{2}+\delta(\mathbf{x})\right]^{q}$ is differentiable, we then obtain

$$
\begin{aligned}
& \left\|E^{j, \ell}\right\|_{L^{2}}^{2}+\frac{\alpha k}{4}\left\|\nabla E^{j, \ell}\right\|_{L^{2}}^{2} \leq C k^{4} h^{-d r}\left\|E^{j, \ell-1}\right\|_{L^{2}}^{2} \\
& \quad+C k^{3} \alpha^{-1}\left[\left\|\left[\left|\nabla U^{j, \ell-2}\right|^{2}+\delta(\mathbf{x})\right]^{\frac{p_{\epsilon}(\mathbf{x})-2}{2}} \nabla E^{j, \ell-1}\right\|_{L^{2}}^{2}\right. \\
& \left.\quad+\max _{\zeta \in\left\{\nabla U^{\left.j, \ell-1, \nabla U^{j, \ell-2}\right\}}\right.}\left\|\frac{p_{\epsilon}(\mathbf{x})-2}{2}\left(|\zeta|^{2}+\delta(\mathbf{x})\right)^{\frac{p_{\epsilon}(\mathbf{x})-4}{2}} 2\left|\zeta\left\|\nabla U^{j, \ell}\right\| \nabla E^{j, \ell-1}\right|\right\|_{L^{2}}^{2}\right] .
\end{aligned}
$$

By an inverse estimate, $\left\|\nabla U^{j, \ell}\right\|_{L^{\infty}} \leq C h^{-\left[\frac{d}{2}+1\right]}\left\|U^{j, \ell}\right\|_{L^{2}}$, and (4.8) we obtain

$$
\begin{aligned}
& \left\|E^{j, \ell}\right\|_{L^{2}}^{2}+\frac{\alpha k}{4}\left\|\nabla E^{j, \ell}\right\|_{L^{2}}^{2} \leq C k^{4} h^{-d r}\left\|E^{j, \ell-1}\right\|_{L^{2}}^{2} \\
& +C k^{3} \alpha^{-1}\left\|\nabla E^{j, \ell-1}\right\|_{L^{2}}^{2}\left[\left.\operatorname{ess}_{\sup }|| \nabla U^{j, \ell-2}\right|^{2}+\left.\delta(\mathbf{x})\right|^{p_{\epsilon}(\mathbf{x})-2}\right.
\end{aligned}
$$

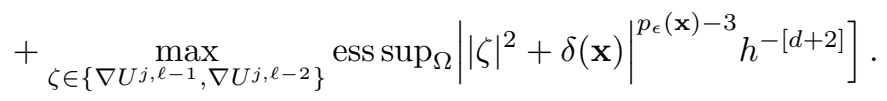

Hence, (4.6) is sufficient to validate a contraction principle for iterates.

Step 2. Overall convergence. The previous step shows that Algorithm $\bar{A}$ terminates. Hence, for every $\theta>0$, and every $j \geq 1$, there exists $\bar{\ell}:=\ell(j, \theta)<\infty$ such that the stopping criterion is met, and for all $W \in V_{h}$, we have

$$
\begin{aligned}
& \left(d_{t}^{2} U^{j}, W\right)+\left(\left[\left|\nabla U^{j}\right|^{2}+\delta(\mathbf{x})\right]^{\frac{p_{\epsilon}(\mathbf{x})-2}{2}} \nabla U^{j}, \nabla W\right)+\left(f\left(\mathbf{x}, U^{j}\right), W\right) \\
& =\left(\left[\left|\nabla U^{j}\right|^{2}+\delta(\mathbf{x})\right]^{\frac{p_{\epsilon}(\mathbf{x})-2}{2}} \nabla U^{j}-\left[\left|\nabla U^{j, \bar{\ell}}\right|^{2}+\delta(\mathbf{x})\right]^{\frac{p_{\epsilon}(\mathbf{x})-2}{2}} \nabla U^{j}, \nabla W\right) \\
& \quad+\left(f\left(\mathbf{x}, U^{j}\right)-f\left(\mathbf{x}, U^{j, \bar{\ell}}\right), W\right) .
\end{aligned}
$$

Because of Step 3 in Algorithm $\bar{A}$, the right-hand side may be bounded by $\theta\left(\|\nabla W\|_{L^{p(\mathbf{x})}}+\|W\|_{L^{2}}\right)$. As a consequence, we may follow the (slightly modified) proof of Theorem 3.1 to conclude subsequence convergence.

Theorem 4.1 specifies situations where Algorithm $\mathrm{A}_{1}$ terminates and motivates combined choices of the regularization function $\delta \in C(\bar{\Omega} ;[0,1])$, the function $p_{\epsilon} \in$ $C(\Omega ;(1, \infty))$, discretization parameters $k, h>0$, and gives a theoretical indication for the stopping criterion (4.4).

\section{Computational experiments}

In this section, we report on experiments where we use Algorithm $\mathrm{A}_{1}$, implemented in MATLAB, with a direct solution of linear systems of equations. Below, let $\mathbf{x}=\left(x_{1}, x_{2}\right)^{\top}$. The function $\left(x_{1}, x_{2}\right) \mapsto \exp \left(-\left(x_{1}-0.5\right)^{2} / 0.03\right) *$ $\exp \left(-\left(x_{2}-0.5\right)^{2} / 0.03\right)$ will be referred to as "hat". All examples use vanishing Neumann boundary conditions, and $\delta=10^{-7}=\theta$. We typically need 2 or 3 fixed-point iterations per step.

The first example is to study the solution's behavior for $p_{+} / p_{-} \gg 1$. It also exemplifies the fact that weak solutions to (1.2) for supercritical growth of $f$ exist, provided initial data satisfy a certain smallness condition.

Example 5.1. Let $\Omega=(0,1)^{2}, T=1, \alpha \in\{0,0.25\}$, as well as $v_{0}=0$, and $u_{0}=2 *$ hat. Choose $f(\mathbf{x}, u)=-|u|^{\gamma-2} u$, for $\gamma=5$, and $p(\mathbf{x})=1+2 x_{1} x_{2}$. 


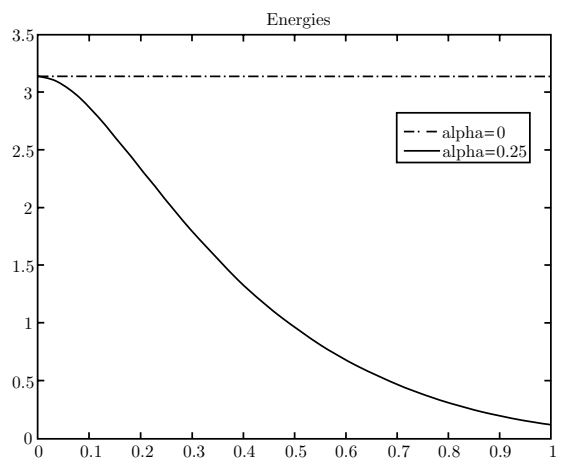

Figure 1. Example 5.1 Energy plot.
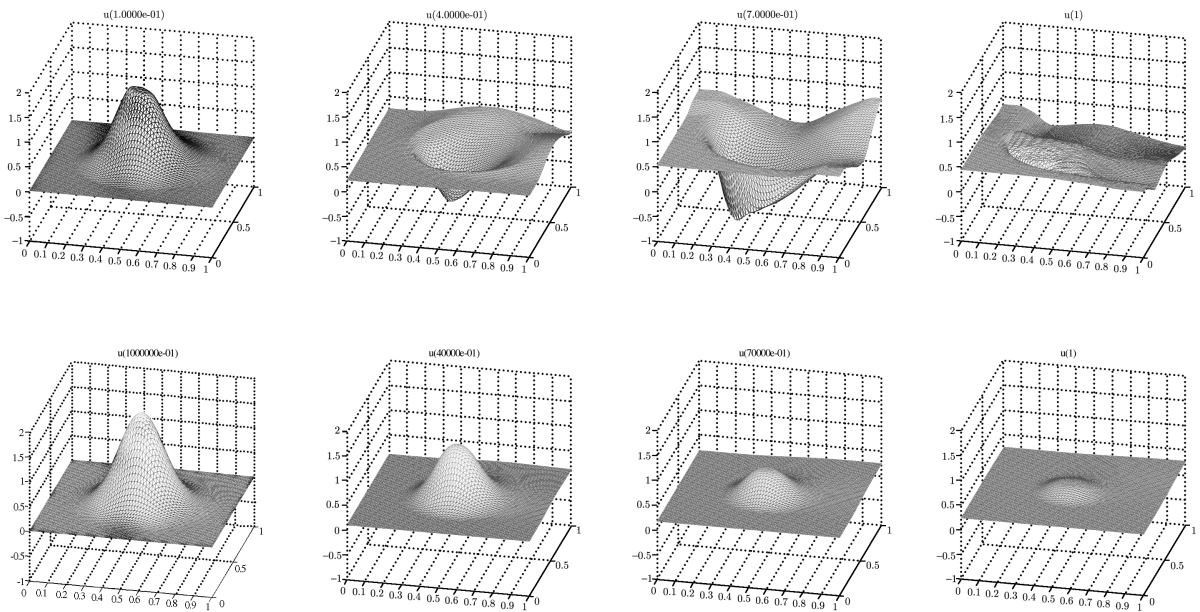

Figure 2. Example 5.1. The two rows show snapshots of $u$ at times $t=0.1,0.4,0.7,1$ (left to right) for $\alpha=0,0.25$, respectively.

Plots for the decaying energy $(\alpha>0)$, resp. constant energy $(\alpha=0)$, are shown in Figure 1. Snapshots of $u$ at times $t=0.1,0.4,0.7,1$ are given in Figure 2 for $\gamma=5$. For $\gamma=1.5$ we experience very similar behavior.

A reverse calculation, started at time $t=T / 2$ for the above example with $\alpha=0$ and $\gamma=1.5$, recovered the initial data up to an $L^{2}$-error of $1.3 * 10^{-3}$, and $L^{\infty}$-error of $5.4 * 10^{-3}$.

The following example is to study $L^{\infty}$-decay of $u, u_{t}$, and $\nabla u$ in time for variable exponents, including a study where $p_{-}=0$.

Example 5.2. Let $\Omega=(0,1)^{2}, T=1, \alpha=1$, as well as $v_{0}=0$, and $u_{0}=0.5 *$ hat. Choose $p_{i}(\mathbf{x})=i+2 x_{1} x_{2}$, for $i \in\{0,1,2\}$, and $f(\mathbf{x}, u)=|u|^{\gamma-2} u$, for $\gamma=6$.

Different decay rates of the energy, as well as the behavior of the $L^{\infty}$-norms of $u, u_{t}$, and $\nabla u$ are shown in Figure 3 for $p_{i}(\mathbf{x})=i+2 x_{1} x_{2}$, with $i \in\{0,1,2\}$.

As the images in the bottom row show, the smaller $p$, the better the initial data are conserved. While $\left\|u_{t}\right\|_{L^{\infty}}$ for $p(\mathbf{x})=2 x_{1} x_{2}$ appears to oscillate, the solution 

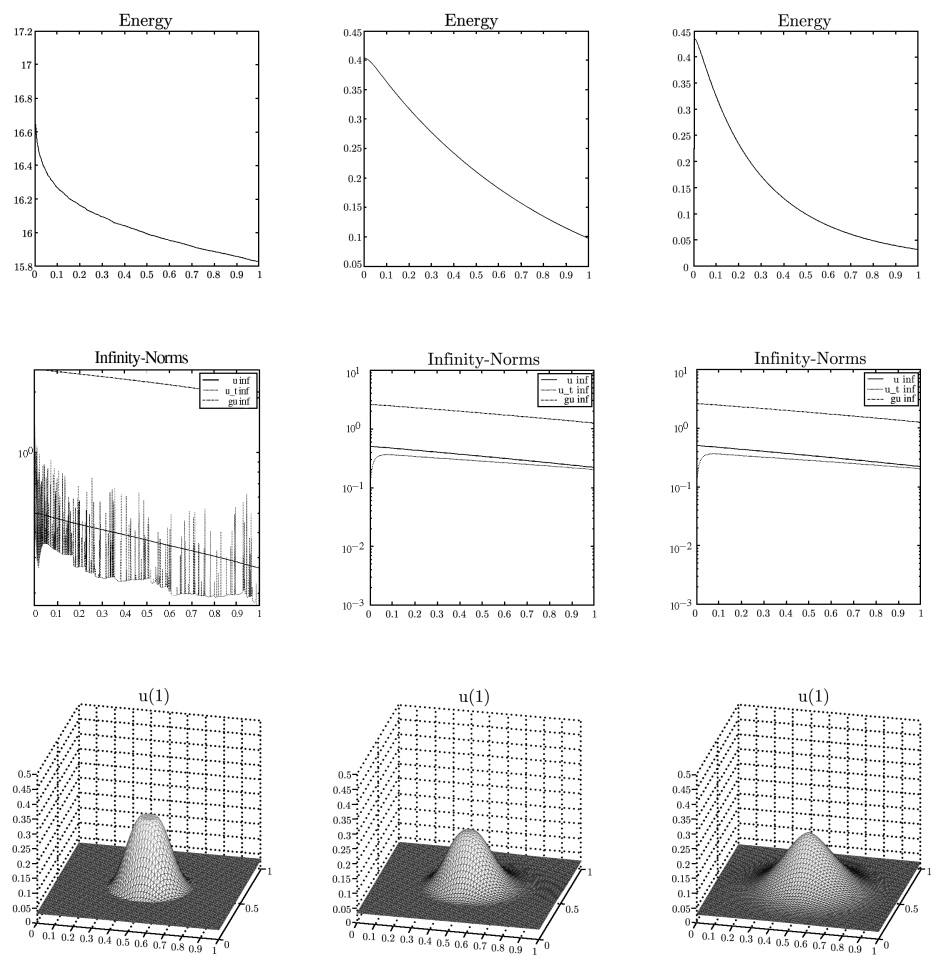

Figure 3. Example 5.2 Top row: Energy decay for $i=0,1,2$. Middle row: $L^{\infty}$-norms of $u, u_{t}$, and $\nabla u$ ( $y$-axis logarithmically scaled) for $i=0,1,2$. Bottom row: $u$ at $T=1$ for $i=0,1,2$.

does not show big changes (note, however, that in this case the logarithmic plot makes the oscillations look worse than they are).

Blowup behavior of (local strong) solutions to (1.2) for $\alpha=0$ has been shown in 22 for nonlinearities where $u f(\mathbf{x}, u) \geq(2+\beta) F(\mathbf{x}, u)$, for some $\beta>0, q \equiv 2$, and $E\left[u_{0}, v_{0}\right]<0$; in [23], it is shown that weak (nonlinear) damping of the form

$$
u_{t t}-\Delta u+\left|u_{t}\right|^{m-2} u_{t}+f(\mathbf{x}, u)=0 \quad \text { in } \Omega_{T}
$$

is insufficient to prevent the blow-up effect in the following sense: for $f(\mathbf{x}, u)=$ $-|u|^{\gamma-2} u$, and $\gamma>m \geq 2$, and negative initial energies, local strong solutions blow up in finite time; i.e., $\lim _{t \rightarrow T^{*}}\|u(t, \cdot)\|_{L^{\infty}}=\infty$, for some $0<T^{*}<\infty$. Moreover, the solution blowup occurs if and only if the energy blows up; i.e., $\lim _{t \rightarrow T^{*}} E_{p}\left[u(t, \cdot), u_{t}(t, \cdot)\right]=-\infty$. (In fact, weak solutions exist if $m \geq \gamma \geq 2$.) In [15], the existence of weak solutions to $(0<\alpha \leq 1$, and constant $p \geq 2)$

$$
u_{t t}-\Delta_{p} u+(\Delta)^{\alpha} u_{t}=|u|^{\gamma-2} u \text { in } \Omega_{T},
$$

for $\gamma \geq p$ is shown, provided that the initial data are properly chosen; blow-up behavior of solutions in the case of negative initial energies and $\gamma>p \geq 2$ is verified in [31, 25, 16].

Examples 5.3 and 5.4 study related questions for variable exponents: blowup for large initial data, and supercritical growth of $f$, for $\alpha=1,0$, respectively. 

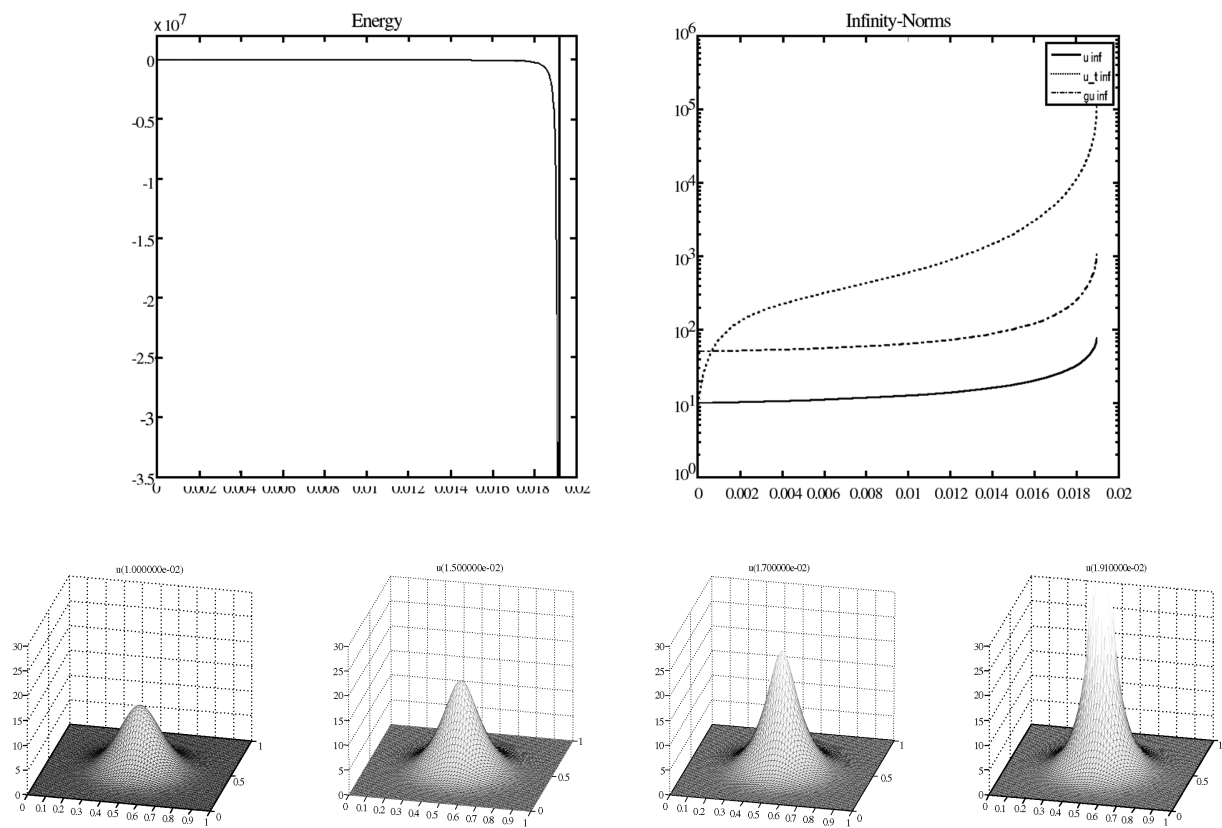

Figure 4. Example 5.3. Top row: Energy (left), and $L^{\infty}$-norms of $u, u_{t}$, and $\nabla u$ (log-plot). Bottom row: Snapshots of $u$ at times $t=0.01,0.015,0.017,0.0191$ (left to right).

Example 5.3. Let $\Omega=(0,1)^{2}, T=1, \alpha=1$, and $u_{0}=10 *$ hat, $v_{0}=0$. Choose $p=2$, and $f(\mathbf{x}, u)=-|u|^{\gamma-2} u$, for $\gamma=6$.

Figure 4 shows energy blowup (to minus infinity), $L^{\infty}$-norms of $u, u_{t}$, and $\nabla u$, as well as snapshots of $u$ at times $t=0.01,0.015,0.017,0.0191$.

This example generalizes the Klein-Gordon equation with focusing nonlinearity, $u_{t t}-\Delta u-u^{5}=0$ in $\Omega_{T}$. In the case of spherically symmetric initial data $\left(u_{0}, v_{0}\right)$ it is known that solutions for small initial data exist and converge to zero [19], and that large data solutions of negative energy blowup in finite time [22]; see also 21]. As is motivated in [7, the static spherically symmetric solution $f(r)=\frac{1}{\sqrt{1+\frac{r^{2}}{3}}}$ $\left(r=\sqrt{x_{1}^{2}+x_{2}^{2}}\right)$ is a candidate for a blowup with unbounded growth at $r=0$ at a rate $\frac{1}{\sqrt{T^{*}-t}}$.

Example 5.4. Let $\Omega=(0,1)^{2}, T=1, \alpha=0$, and $u_{0}=3 *$ hat, $v_{0}=0$. Choose $p=3-2 *$ hat, and $f(\mathbf{x}, u)=-|u|^{\gamma-2} u$, for $\gamma=6$.

Figure 5 shows energy blowup (apparently to plus infinity, but we are not sure whether this is a numerical artifact), $L^{\infty}$-norms of $u, u_{t}$, and $\nabla u$, as well as snapshots of $u$ at times $t=0.05,0.1,0.15,0.154$.

Solutions to both numerical schemes as well as the PDE (1.2) use energy-based arguments, which require initial data of finite energy, in particular. In our last example, we evolve initial data of low regularity/infinite energy, which leads to interesting issues concerning locally existing solutions of typical nonlinear equations [24, as well as blowup, as shown in the next example. 

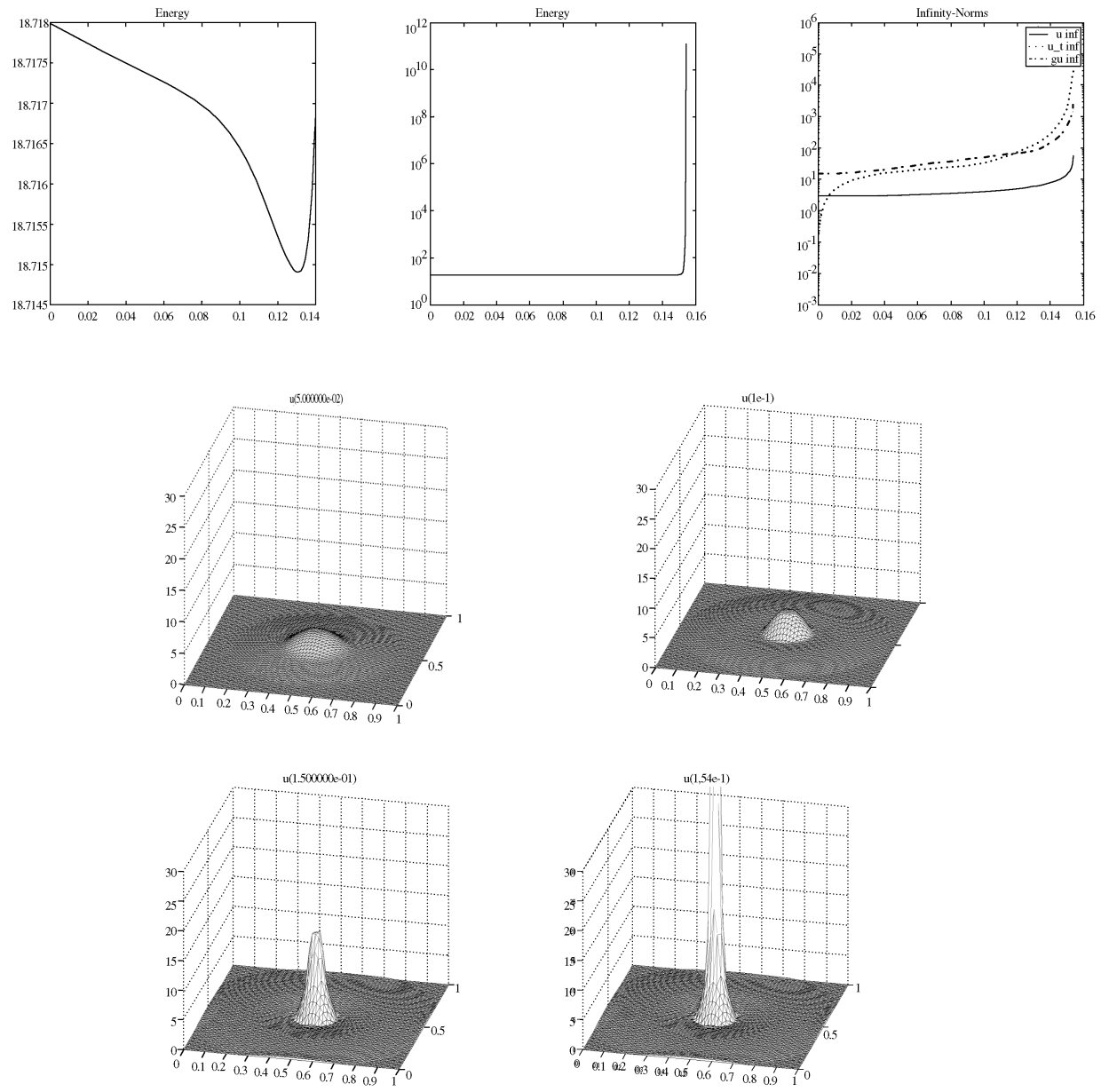

Figure 5. Example 5.4. Top row: Energy before blowup (left), energy including blowup (middle, log-plot), and $L^{\infty}$-norms of $u$, $u_{t}$, and $\nabla u$ (log-plot). Middle and bottom rows: Snapshots of $u$ at times $t=0.05,0.1,0.15,0.154$.

Example 5.5. Let $\Omega=(0,1)^{2}, T=1, \alpha=0, v_{0}=0$, and $u_{0}=5$ on $[0.25,0.375]^{2} \cup$ $[0.625,0.75]^{2}$, and zero elsewhere. Let $f(\mathbf{x}, u)=|u|^{\gamma-2} u$, for $\gamma=6$, and $p(\mathbf{x})=$ $1+2 x_{1} x_{2}$.

Figure 6 shows energy blowup (to plus infinity) for different space discretizations $h=1 / 16,1 / 32,1 / 64$. The qualitative behavior of the solutions for different $h$ seems to be the same. Note, however, that the initial energy for $h=1 / 16$ is negative, while it is positive for finer $h . L^{\infty}$-norms of $u, u_{t}$, and $\nabla u$, as well as snapshots of $u$ at times $t=0.001,0.04,0.046,0.0486$ are given for the finest $h$ only.

Again, we see (compare front and rear bricks) that (locally) smaller $p$ better preserve the structure, while with (locally) larger $p$, the brick crumbles much more. Nevertheless, the large right-hand side ensures that both bricks blow up. 

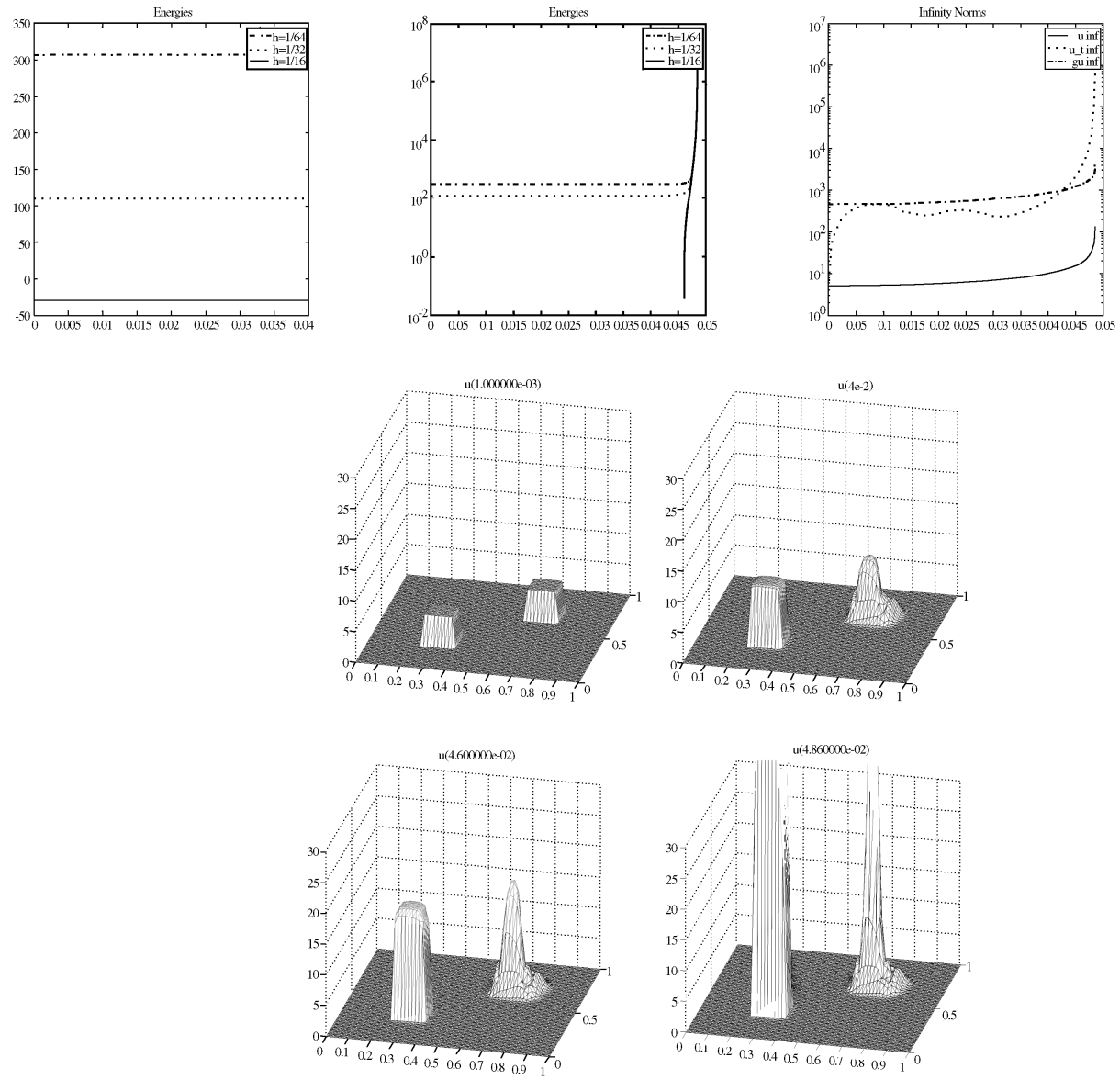

Figure 6. Example 5.5. Top row: Energies before blowup (left), energies including blowup (middle, log-plot) for different space discretizations $h=1 / 16,1 / 32,1 / 64$, as well as $L^{\infty}$-norm plots of $u$, $u_{t}$, and $\nabla u$ (log-plot) for $h=1 / 64$. Middle and bottom rows: Snapshots for $h=1 / 64$ and $t=0.001,0.04,0.046,0.0486$.

Additional examples and short movies of the above computational studies can be found online at http://na.uni-tuebingen.de/ haehnle/wpx/.

\section{REFERENCES}

1. Stanislav N. Antontsev and Sergei I. Shmarev, Anisotropic parabolic equations with variable nonlinearity, Preprint, URL: http://cmaf.ptmat.fc.ul.pt/preprints/preprints.html, 2007.

2. _ Parabolic equations with anisotropic nonstandard growth conditions, Free boundary problems, Internat. Ser. Numer. Math., vol. 154, Birkhäuser, Basel, 2007, pp. 33-44. MR2305342 (2007m:35118)

3. Hajer Bahouri and Jalal Shatah, Decay estimates for the critical semilinear wave equation, Ann. Inst. H. Poincaré Anal. Non Linéaire 15 (1998), no. 6, 783-789. MR1650958|(99h:35136)

4. John MacLeod Ball, Remarks on blow-up and nonexistence theorems for nonlinear evolution equations, Quart. J. Math. 28 (1977), no. 4, 473-486. MR0473484(57:13150) 
5. _ Global attractors for damped semilinear wave equations, Discrete Contin. Dyn. Syst. 10 (2004), no. 1-2, 31-52, Partial differential equations and applications. MR2026182 (2005a:37149)

6. Abbès Benaissa and Soufiane Mokeddem, Decay estimates for the wave equation of pLaplacian type with dissipation of m-Laplacian type, Math. Methods Appl. Sci. 30 (2007), no. 2, 237-247. MR2285123 (2007k:35326)

7. Piotr Bizoń, Threshold behavior for nonlinear wave equations, J. Nonlinear Math. Phys. 8 (2001), no. suppl., 35-41, Nonlinear evolution equations and dynamical systems (Kolimbary, 1999). MR 1821505

8. Piotr Bizoń, Tadeusz Chmaj, and Zbisław Tabor, On blowup for semilinear wave equations with a focusing nonlinearity, Nonlinearity 17 (2004), no. 6, 2187-2201. MR2097671 (2005f:35210)

9. Michel Crouzeix and Vidar Thomée, The stability in $L_{p}$ and $W_{p}^{1}$ of the $L_{2}$-projection onto finite element function spaces, Math. Comp. 48 (1987), no. 178, 521-532. MR878688 (88f:41016)

10. Lars Diening, Lebesgue and Sobolev spaces with variable exponent, 2007, Habilitation Thesis.

11. Xian-Ling Fan and Qi-Hu Zhang, Existence of solutions for $p(x)$-Laplacian Dirichlet problem, Nonlinear Anal. 52 (2003), no. 8, 1843-1852. MR1954585 (2004f:35060)

12. Xianling Fan and Dun Zhao, On the generalized Orlicz-Sobolev space $W^{k, p(x)}(\Omega)$, J. Gansu Educ. College 12 (1998), no. 1, 1-6.

13. On the spaces $L^{p(x)}(\Omega)$ and $W^{m, p(x)}(\Omega)$, J. Math. Anal. Appl. 263 (2001), no. 2, 424-446. MR1866056 (2003a:46051)

14. Victor A. Galaktionov and Stanislav I. Pohozaev, Blow-up, critical exponents and asymptotic spectra for nonlinear hyperbolic equations, Preprint, URL: ftp://ftp.maths.bath.ac.uk/ pub/preprints/maths0010.ps.Z, 2000.

15. Hongjun Gao and To $\mathrm{Fu} \mathrm{Ma}$, Global solutions for a nonlinear wave equation with the $p$ Laplacian operator, Electron. J. Qual. Theory Differ. Equ. (1999), no. 11. MR1718343 (2000j:35195)

16. Hongjun Gao and Hui Zhang, Global nonexistence of the solutions for a nonlinear wave equation with the q-Laplacian operator, J. Partial Differential Equations 20 (2007), no. 1, 71-79. MR2317126 (2008a:35195)

17. Vladimir Georgiev and Grozdena Todorova, Existence of a solution of the wave equation with nonlinear damping and source terms, J. Differential Equations 109 (1994), no. 2, $295-308$. MR1273304 (95b:35141)

18. Ryo Ikehata, Tokio Matsuyama, and Mitsuhiro Nakao, Global solutions to the initial-boundary value problem for the quasilinear viscoelastic wave equation with a perturbation, Funkcial. Ekvac. 40 (1997), no. 2, 293-312. MR1480280(99h:35141)

19. Fritz John, Blow-up of solutions of nonlinear wave equations in three dimensions, Manuscripta Math. 28 (1979), 235-268. MR.535704 (80i:35114)

20. Varga K. Kalantarov and Olga A. Ladyzhenskaya, The occurrence of collapse for quasilinear equations of parabolic and hyperbolic types, J. Soviet Math. 10 (1978), 53-70. MR0604036 $(58: 29269)$

21. Carlos E. Kenig and Frank Merle, Global well-posedness, scattering and blowup for the energy critical focusing non-linear wave equation, Preprint, URL: http://arxiv.org/abs/math.AP/0610801, 2007.

22. Howard A. Levine, Instability and nonexistence of global solutions to nonlinear wave equations of the form $P u_{t t}=-A u+\mathcal{F}(u)$, Trans. Amer. Math. Soc. 192 (1974), 1-21. MR0344697 $(49: 9436)$

23. Howard A. Levine and Grozdena Todorova, Blow up of solutions of the Cauchy problem for a wave equation with nonlinear damping and source terms and positive initial energy, Proc. Amer. Math. Soc. 129 (2001), no. 3, 793-805 (electronic). MR.1792187 (2001k:35212)

24. Hans Lindblad, Counterexamples to local existence for nonlinear wave equations, Journées "Équations aux Dérivées Partielles" (Saint-Jean-de-Monts, 1994), École Polytech., Palaiseau, 1994, Exp. No. X, 5 pp. MR.1298681 (95k:35136)

25. Salim A. Messaoudi and Belkacem Said Houari, Global non-existence of solutions of a class of wave equations with non-linear damping and source terms, Math. Methods Appl. Sci. 27 (2004), no. 14, 1687-1696. MR2089156(2005e:35169) 
26. Mitsuhiro Nakao, Energy decay for the quasilinear wave equation with viscosity, Math. Z. 219 (1995), no. 2, 289-299. MR1337222 (96d:35092)

27. Stanislav Pohozaev and Laurent Véron, Blow-up results for nonlinear hyperbolic inequalities, Ann. Scuola Norm. Sup. Pisa Cl. Sci. (4) 29 (2000), no. 2, 393-420. MR 1784180 (2001m:35227)

28. Andreas Prohl, Convergence of a finite element-based space-time discretization in elastodynamics, SIAM Journal on Numerical Analysis 46 (2008), no. 5, 2469-2483. MR2421043

29. Andreas Prohl and Isabelle Weindl, Convergence of an implicit finite element discretization for a class of parabolic equations with nonstandard anisotropic growth conditions, Preprint, URL: http://na.uni-tuebingen.de/preprints.shtml, 2007.

30. Stefan G. Samko, On a progress in the theory of Lebesgue spaces with variable exponent: Maximal and singular operators, Integral Transforms Spec. Funct. 16 (2005), no. 5-6, 461482. MR2138062 (2005k:42056)

31. Zhijian Yang, Blow-up of solutions for a class of non-linear evolution equations with nonlinear damping and source terms, Math. Meth. Appl. Sci. 25 (2002), 825-833. MR1910734 (2003f:35217)

32. Qihu Zhang, Existence of blow-up solutions to a class of $p(x)$-Laplacian problems, Int. J. Math. Anal. (Ruse) 1 (2007), no. 1-4, 79-88. MR2340930 (2008e:35080)

33. Dun Zhao, W. J. Quang, and Xian Ling Fan, On generalized Orlicz spaces $L^{p(x)}(\Omega)$, J. Gansu Sci. 9 (1996), no. 2, 1-7.

Mathematisches Institut, Universität Tübingen, Auf der Morgenstelle 10, D-72076 Tübingen, Germany

E-mail address: haehnle@na.uni-tuebingen.de

Mathematisches Institut, Universität TüBingen, Auf Der Morgenstelle 10, D-72076 Tübingen, Germany

E-mail address: prohl@na.uni-tuebingen.de 